\title{
Stochastic Geometry Modeling of Cellular Uplink Power Control under Composite Rayleigh-Lognormal Fading
}

\author{
Prasanna Herath*†, Chintha Tellambura*, and Witold A. Krzymien ${ }^{* \dagger}$ \\ *University of Alberta, Edmonton \\ ${ }^{\dagger}$ Telecommunications Research Laboratories, Edmonton \\ Email: prasanna@ualberta.ca, \{chintha,wak\}@ece.ualberta.ca
}

\begin{abstract}
Power control for uplink transmission in a randomly laid-out cellular network operating over an environment with path loss and composite Rayleigh-lognormal shadowing is investigated. Each mobile station (MS) adjusts its transmit power to completely remove shadowing and to partially invert the effect of path loss. Using stochastic geometry tools, we derive an approximate coverage probability expression and validate it via simulations. With the power control scheme considered, shadowing with lower standard deviation improves the coverage probability. Also the severity of shadowing of local and surrounding environments has the same effect on the coverage probability. It was also observed that at low signal-to-interference-plus-noise ratio (SINR) thresholds, complete compensation of shadowing and partial compensation of path loss improves coverage, while at high SINR thresholds inverting only the effect of shadowing gives a better coverage probability.
\end{abstract}

\section{INTRODUCTION}

In cellular networks, transmit power control plays a major role in the management of interference, energy, and connectivity [1], [2]. Compared to the downlink, uplink power control is of paramount importance for two reasons. (i) The downlink transmissions all originate from cell centers, whereas uplink transmissions can come from cell boundaries [1]. Further, the demand based deployment of base stations (BSs) has made modern cellular networks more irregular and complex than their predecessors. Therefore, even when a mobile station (MS) is associated with the BS providing the highest average signal strength, interference from another MS can be much stronger than the desired signal at the BS [3]. (ii) Batterypowered MSs need to be very power-efficient. Uplink power control thus aims at improving the cell edge user experience and power efficiency of mobile devices.

In this paper, stochastic geometry tools are used to investigate the effect of transmit power control on the uplink of cellular networks under composite Rayleigh-lognormal fading. A fractional power control (FPC) scheme, which compensates for the path loss and large scale shadowing, is considered. The Poisson point process (PPP) [4] is used to characterize the spatial distribution of MSs and BSs. The use of PPP results in simple and accurate expressions, which helps to characterize and understand the network behavior [5]-[7].

\section{A. Prior related research}

Uplink coverage probability and average rate with FPC, where each MS changes its transmit power to partially invert the effect of path loss, are investigated in [6]. Single tier BSs with closest-BS association, power-law path loss model and Rayleigh fading are considered. The coupling between the locations of BSs and associated MSs is captured by modeling MSs by a homogeneous PPP and assuming that the serving BS of each MS is uniformly distributed in the Voronoi cell [4] of the MS. Further, the MS-BS distances are assumed to be independent and identically distributed (i.i.d.) with the distribution of distance between any BS and its closest MS on $\mathbb{R}^{2}$. In [6], it is shown that power control in the form of partial channel inversion should be used at low signal-to-interference-plusnoise ratio (SINR), while full power transmission is optimal at higher SINR. The outage probability and spectral efficiency of the uplink of single-tier cellular networks with truncated channel-inversion power control is investigated in [3]. In this scheme, each MS associates with the closest BS and adjusts its transmit power such that a predefined average received power level (after averaging over shadowing and multipath fading) is maintained at its serving BS provided that the required transmit power does not exceed the maximum transmit power of MSs. When this is not possible, MSs do not transmit. This analysis is extended to multi-tier cellular networks in [7]. Reference [8] investigates the uplink capacity in a two-tier direct sequence code division multiple access (DS-CDMA) cellular network consisting of macro BSs and femto access points (APs). A power control scheme which compensates for path loss and log-normal shadowing is considered.

\section{B. Motivation and our contribution}

In most previous uplink analyses, either power control is ignored or limited to compensating path loss only. However, compared to traditional macro BSs, antenna heights of small BSs of modern cellular networks, for example pico BSs, is considerably lower. Thus the uplink transmission will be subject to significant shadowing. Therefore, power controlling schemes alleviating shadowing in addition to path loss will undoubtedly improve the coverage, data rates, and power efficiency of these networks. Reference [8] also investigates the cellular uplink with power control based on path loss and lognormal shadowing. However, it investigates a two-tier DSCDMA cellular network, while this paper is focused on a cellular network using orthogonal frequency division multiple access (OFDMA) or DFT precoded OFDMA (SC-FDMA).

Consequently, we consider an FPC scheme, which not only partially compensates the effect of path loss, but also 
completely inverts the effect of shadowing. Moreover, since many previous studies are limited to small scale fading while ignoring shadowing, we consider more practical composite Rayleigh-lognormal fading. With this power control, the transmit power of an MS becomes random depending on MS-BS distance and channel gain due to shadowing. Using stochastic geometry tools, we derive approximate expressions for the probability density function (PDF) of the MS transmit power and the coverage probability and validate via simulations. These expressions help us to investigate the effects of power control and shadowing on the coverage probability.

Notations: $f_{X}(\cdot)$ and $F_{X}(\cdot)$ represent the PDF and cumulative distribution function $(\mathrm{CDF})$ of random variable $X . \mathbb{E}[\cdot]$ is the expectation. The Laplace transform of the PDF of a random variable $X$ is denoted as $\mathcal{L}_{X}(s)$.

\section{SySTEM MODEL}

We consider uplink transmission in a cellular network with transmit power control. A single class of BSs, micro BSs for example, is assumed. The spatial distribution of MSs is modelled by a homogeneous PPP $\Phi$ of intensity $\lambda$ over $\mathbb{R}^{2}$. This assumption has recently been widely used to characterize the spatial distribution of MSs and BSs [5]-[7], [9]. We assume that each MS associates with the BS providing the highest signal strength after averaging out variations due to shadowing and multipath fading, which is equivalent to connecting to the closest BS.

Universal frequency reuse is assumed with OFDMA or SCFDMA transmission. A fully loaded network is assumed, i.e., each BS has an active uplink transmission scheduled for each time-frequency resource block. With this network setup, it is reasonable to assume that each BS is uniformly distributed in the Voronoi cell of its corresponding MS [6]. We investigate the coverage probability of an MS located at $x \in \Phi$. Since a homogeneous PPP in $\mathbb{R}^{2}$ is translation and rotation invariant, without loss of generality we can assume the BS associated with $x$ to be located at the center of the network. Similarly to [10], the coverage probability is defined as the probability that a randomly chosen MS achieves a target SINR $T$.

Power-law path loss and composite Rayleigh-lognormal fading are assumed. Since the severity of lognormal shadowing depends on the density and locations of shadowing objects, it can be different from one cell to another. However, considering different shadowing conditions for each cell is mathematically intractable. Therefore, we assume only two different shadowing standard deviations: one for the local environment (locality of the BS serving MS $x$ ), $\xi$, and another for interfering cells, $\sigma . \alpha$ and $\nu>2$ represent the path loss exponents of the desired and interfering signals, respectively. The condition $\nu>2$ is to maintain a finite interference power at each BS. In the FPC scheme considered in this paper the effect of path loss is partially compensated, while the effect of shadowing is completely eliminated. This FPC scheme is described next.

\section{A. Uplink Power Control}

Consider an MS located at distance $r$ from its serving BS. The power control aims to partially compensate the effect of path loss on the received signal power at the BS and fully compensate for the channel gain/attenuation due to shadowing. Therefore, the transmit power at the MS can be written as

$$
P=\frac{\rho r^{\alpha \eta}}{h}
$$

where $h$ is the channel power gain due to lognormal shadowing. $h \sim \operatorname{Lognormal}(0, \xi)$ for $\operatorname{MS} x^{1}$ and $h \sim \operatorname{Lognormal}(0, \sigma)$ for MSs $z \in \Phi \backslash x . \eta \in[0,1]$ is the power control factor, which determines to what extent the effect of path loss is compensated by the power control scheme. $\eta=1$ gives a complete inversion of path loss. $\eta=0$ represents the case where only shadowing is inverted. $\rho$ is a constant, which can be selected such that a pre-determined mean target received power level (after variation due to multipath fading is averaged out) $P_{\mathrm{e}}$ is maintained by cell-edge users. Assuming circular cells, average distance between a cell-edge user and its serving BS can be written as $R_{\mathrm{e}}=1 / \sqrt{\pi \lambda}$. Therefore, $\rho$ can be computed as $\rho=\frac{P_{e}}{R_{e}^{\alpha(\eta-1)}}=P_{e}(\pi \lambda)^{\frac{\alpha}{2}(\eta-1)}$.

\section{TRAnsmit Power and Coverage Probability ANALYSIS}

The uplink analysis is more complicated because of the coupling between the locations of MSs and their associated BSs. This is because of the restriction of one MS per resource block in a given cell. Further the use of power control to overcome channel impairments such as path loss and shadowing makes the transmit powers of different MSs highly variable and correlated. In our analysis, two assumptions (Assumption 1 and 2) are used to overcome these challenges. Using Assumption 1, first we derive an approximation for the PDF of transmit power $P$. Subsequently, the coverage probability is derived using Assumption 2. In Section IV we show that the loss of accuracy of analytical results due to these assumptions is minimal.

\section{A. Transmit Power Analysis}

Due to the random network topology and transmit power control, each MS will transmit with power given by (1). We use the following assumption to obtain the PDF of the transmit power of an MS,

Assumption 1: According to the system model (Section II), BS is uniformly distributed in the Voronoi cell of the MS being served. We approximate this choice of BS by a point uniformly chosen in $\mathbb{R}^{2}$. Under this assumption, the distance between an MS and its associated BS follows the Rayleigh PDF given by

$$
f_{r}(r)=2 \pi \lambda r \exp \left(-\pi \lambda r^{2}\right), \quad 0 \leq r \leq \infty .
$$

The same assumption is made in [6]. Using PDF in (2), we derive the PDF of the transmit power of an MS given in the following lemma.

\footnotetext{
${ }^{1}$ With a slight abuse of notation we will use $\mathrm{x}$ to denote both the location of MS and MS itself. Similarly for $z$.
} 
Lemma 1: In a Poisson cellular network with shadow inverted FPC, PDF of the transmit power of an MS can be approximated by

$$
\begin{aligned}
& f_{P}(z) \approx \frac{2 \sqrt{\pi} \lambda}{\alpha \eta \rho^{2 / \alpha \eta}} \sum_{i=1}^{N} w_{i} e^{\frac{2 \sqrt{2} \sigma u_{i}}{\alpha \eta}} z^{\frac{2}{\alpha \eta}-1} \\
& \quad \exp \left[-\frac{\pi \lambda}{\rho^{2 / \alpha \eta}} z^{2 / \alpha \eta} e^{\frac{2 \sqrt{2} \sigma u_{i}}{\alpha \eta}}\right], \quad 0 \leq z \leq \infty,
\end{aligned}
$$

where $N$ is an integer which determines the accuracy of the approximation. The weights $w_{i}$ and abscissas $u_{i}$ are determined by Hermite polynomial after $N$ is chosen. $w_{i}$ and $u_{i}$ for different $N$ values are available in [11, Table (25.10)] or can be calculated by a simple MATLAB ${ }^{\circledR}$ program.

Proof: See Appendix A.

\section{B. Coverage Probability Analysis}

Without loss of generality, we assume that the BS associated with the MS under investigation $x \in \Phi$ is located at the center of $\mathbb{R}^{2}$. SINR at this BS can be written as

$$
\mathrm{SINR}=\frac{\rho\|x\|^{\alpha(\eta-1)} g}{\sum_{z \in \Phi \backslash x} P_{z}\|z\|^{-\nu} h_{z}+N_{0}},
$$

where $g \sim \exp (1)$ is the power gain of the serving BS MS channel subject to Rayleigh multipath fading. $P_{z}$ is the transmit power of interfering MS $z \in \Phi \backslash x$. $\|z\|$ and $\|x\|$ are the Euclidean distances from MSs $z$ and $x$ to the BS located at the center of $\mathbb{R}^{2}$, respectively. The variance of the additive white Gaussian noise is given by $N_{0} . h_{z}$ is the power gain of the interfering MS $z$ - serving BS channel subject to composite Rayleigh-lognormal fading. PDF of $h_{z}$ is given by

$$
f_{h_{z}}(h)=\int_{0}^{\infty} \frac{1}{\mu} e^{\frac{-h}{\mu}} \frac{e^{-\frac{(\ln (\mu))^{2}}{2 \xi^{2}}}}{\sqrt{2 \pi} \xi \mu} d \mu .
$$

It should be noted that transmit powers $P$ of $x, z \in \Phi$ are identically distributed, but not independent in general. This is due to the dependency in the distance between MSs and their serving BSs introduced by the Voronoi tessellation and the restriction that only one BS is located in each Voronoi cell. However, in [6], it has been shown that this dependency is weak, and therefore can be ignored. This independence assumption is formerly stated next.

Assumption 2: We assume that transmit powers $P$ of $x, z \in$ $\Phi$ are statistically independent.

With Assumption 2, $P_{z}, z \in \Phi \backslash x$ are i.i.d. with PDF given by (3). We use assumptions 1 and 2 to investigate the coverage probability of the MS being served by the BS located at the center of the network. Coverage probability is formerly stated in the following theorem.

Theorem 1: The uplink coverage probability can be approximated by:

$P_{c} \approx 2 \pi \lambda \int_{0}^{\infty} r_{x} e^{-\frac{T N_{0} r_{x}^{\alpha}(1-\eta)}{\rho}-\pi \lambda r_{x}^{2}} \mathcal{L}_{I_{\Phi \backslash x}}\left(\frac{\operatorname{Tr}_{x}^{\alpha(1-\eta)}}{\rho}\right) d r_{x}$, where $I_{\Phi \backslash x}=\sum_{z \in \Phi \backslash x} P_{z}\|z\|^{-\nu} h_{z}$ is the total interference power at the BS located at the center of $\mathbb{R}^{2}$.

Proof: See Appendix B.

The Laplace transform of the PDF of $I_{\Phi \backslash x}, \mathcal{L}_{I_{\Phi \backslash x}}(s)$ can be derived as

$$
\begin{aligned}
& \mathcal{L}_{I_{\Phi \backslash x}}(s) \approx \exp \left(-2 \pi \sum_{i=1}^{N} w_{i} \sum_{j=1}^{M} \beta_{j} \sum_{k}^{L} \kappa_{k}\right. \\
&\left.\int_{r_{x}}^{\infty} \frac{r_{z}}{1+\frac{r_{z}^{\nu}(\pi \lambda)^{\frac{\alpha \eta}{2}} \exp \left(\sqrt{2} \sigma u_{i}\right)}{s \rho \exp \left(\sqrt{2} \xi t_{j}\right) x_{k}^{\frac{\alpha \eta}{2}}}} d r_{z}\right) .
\end{aligned}
$$

Proof: See Appendix C.

\section{NUMERICAL RESULTS}

This section presents numerical and simulation results and investigates the effect of standard deviations of shadowing and power control factor on the coverage probability of the networks. Since natural logarithm is used instead of logarithm with base 10, PDFs (5) and (9) are scaled versions of the actual PDFs [12]. Therefore, the relationships of the standard deviations of shadowing in $\mathrm{dB} \sigma_{\mathrm{dB}}$ and $\xi_{\mathrm{dB}}$ given in Figs. 1,2 , and 3 , can be written as $\sigma_{\mathrm{dB}}=(10 / \ln 10) \sigma \mathrm{dB}$ and $\xi_{\mathrm{dB}}=(10 / \ln 10) \xi \mathrm{dB}$.

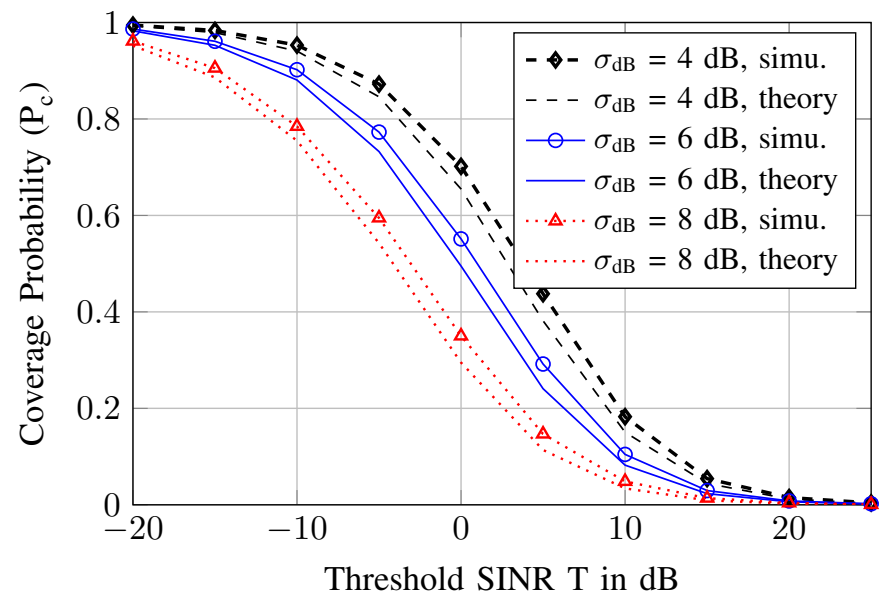

Fig. 1: Coverage probability vs SINR threshold for different values of shadow fading standard deviation. $\eta=0.5 . \lambda=$ $1 \mathrm{BS} \mathrm{km}^{-2}, \alpha=3.5, \nu=3.7, N_{0}=0, \xi_{\mathrm{dB}}=\sigma_{\mathrm{dB}}, \rho=-80$ $\mathrm{dBm}$.

Fig. 1 shows the variation of coverage probability with the threshold SINR for various degrees of shadowing. It shows that Theorem 1 closely matches the simulation results. Lower $\sigma_{\mathrm{dB}}$ (less severe shadowing) improves the coverage probability. This is because the power control scheme completely removes the effect of shadowing on the desired signal, but the effect of shadowing on interfering signals remains unchanged.

Fig. 2 shows the variation of coverage probability with power control factor $\eta$. When $\eta=1$, both path loss and shadowing are fully compensated for, resulting a constant 


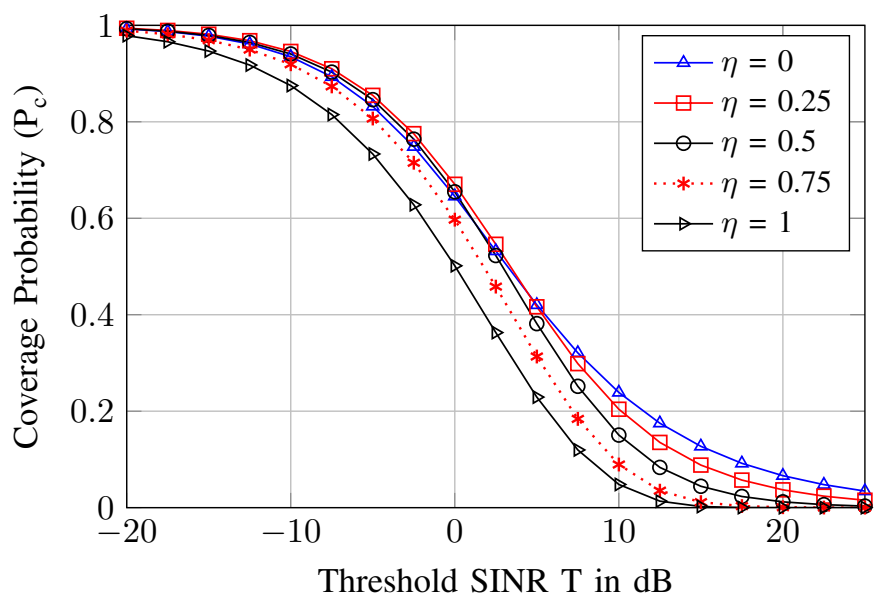

Fig. 2: Coverage probability vs SINR threshold for different values of power control factor $\eta . \lambda=1 \mathrm{BS} \mathrm{km}^{-2}, \alpha=3.5$, $\nu=3.7, N_{0}=0, \sigma_{\mathrm{dB}}=\xi_{\mathrm{dB}}=4 \mathrm{~dB}, \rho=-80 \mathrm{dBm}$.

received power level $\rho$ at the serving BS for all the MSs regardless of their location. On the other hand $\eta=0$ only compensates for shadowing. The case $\eta=0$ does not always provide the highest coverage probability, but provides higher coverage probability for higher SINR thresholds. Also as $\eta$ increases above 0.5 , the coverage probability drops considerably. Therefore, we can conclude that at low SINR thresholds complete elimination of shadow fading and partial compensation of path loss gives better coverage, while at high SINR thresholds inverting only the effect of shadowing gives a better coverage probability.

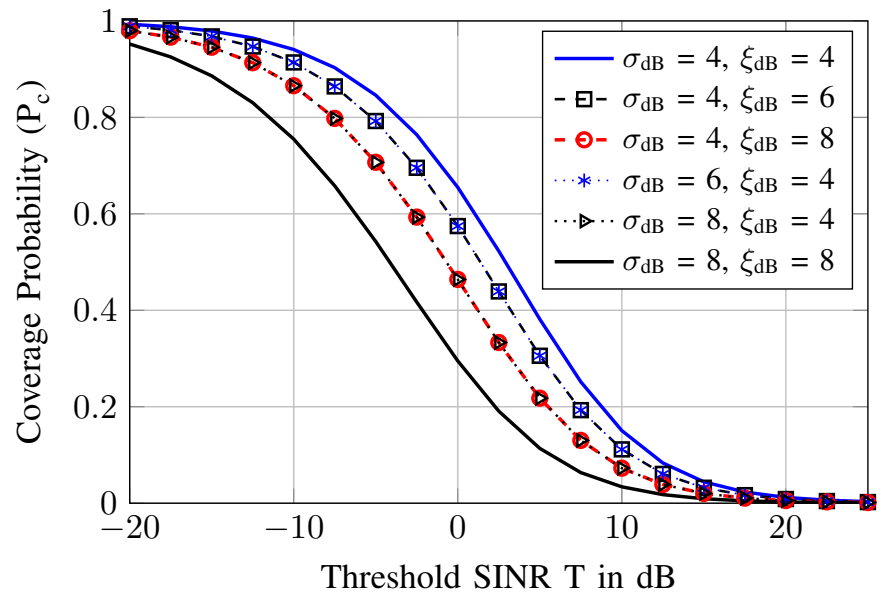

Fig. 3: Coverage probability vs SINR threshold for different shadow fading environments, $\eta=0.5$. $\lambda=1 \mathrm{BS} \mathrm{km}^{-2}, \alpha=$ $3.5, \nu=3.7, N_{0}=0, \mathrm{~dB}, \rho=-80 \mathrm{dBm}$.

Fig. 3 shows the coverage probability under different shadowing environments. Previous studies have shown that the standard deviation of shadow fading is nearly independent of radio path length [12]. $\xi_{\mathrm{dB}}$ represents the shadowing environment of $\mathrm{BS}$ under investigation, while $\sigma_{\mathrm{dB}}$ represents shadowing environment in cells of interfering MSs. Thus a lower $\xi_{\mathrm{dB}}$ compared to $\sigma_{\mathrm{dB}}$ represents the case where BS under investigation is located in a less severely shadowed environment compared to cells of interfering MSs. Interestingly, results show that the shadowing standard deviations of local and surrounding environments have the same effect on the coverage probability under the power control scheme considered in this paper. For example, coverage probability of the network with $\sigma_{\mathrm{dB}}=4 \mathrm{~dB}, \xi_{\mathrm{dB}}=6 \mathrm{~dB}$ is similar to that of the networks with $\sigma_{\mathrm{dB}}=6 \mathrm{~dB}, \xi_{\mathrm{dB}}=4 \mathrm{~dB}$.

\section{CONCLUSION}

Uplink power control for a cellular network operating over path loss and composite Rayleigh-lognormal fading has been investigated. Each MS adjusts its transmit power to partially compensate for path loss and fully compensate for lognormal shadowing. Approximate expressions have been derived for the transmit power PDF and the coverage probability, and validated via Monte-Carlo simulations. It was observed that when a proper power control scheme is in use, shadowing with lower standard deviation (less severe shadowing) improves the coverage probability of cellular systems. Further, it was observed that, at low SINR complete elimination of shadow fading and partial compensation of path loss gives better coverage probability, while at high SINR inverting only the effect of shadowing gives a better coverage probability. It was also observed that with the proposed power control scheme, change of the shadowing standard deviation of local or surrounding environments has the same effect on the uplink coverage probability.

\section{APPENDIX A}

PDF of the transmit power at an MS can be derived as follows. Since each MS transmits with power given by (1)

$$
\begin{aligned}
F_{P}(z) & =\operatorname{Pr}[P<z]=\int_{0}^{\infty} \operatorname{Pr}\left[r<\left(\frac{z h}{\rho}\right)^{\frac{1}{\alpha \eta}} \mid h=t\right] f_{h}(t) d t \\
& \stackrel{a}{\approx} \int_{0}^{\infty}\left[1-e^{-\pi \lambda\left(\frac{z t}{\rho}\right)^{2 / \alpha \eta}}\right] f_{h}(t) d t
\end{aligned}
$$

where step (a) follows from Assumption 1. The PDF of lognormal fading coefficient $h$ is given by

$$
f_{h}(t)=\frac{1}{\sqrt{2 \pi} \sigma t} e^{\frac{-(\ln t)^{2}}{2 \sigma^{2}}}, \quad 0<t<\infty
$$

Substituting (9) in (8), and using the change of variable $u=$ $\frac{\ln (t)}{\sqrt{2} \sigma}$

$$
F_{P}(z) \approx 1-\frac{1}{\sqrt{\pi}} \int_{-\infty}^{\infty} e^{-\pi \lambda\left(\frac{z}{\rho}\right)^{2 / \alpha \eta}+e^{\frac{2 \sqrt{2} \sigma u}{\alpha \eta}}} e^{-u^{2}} d u
$$

(10) has the form of Hermite integration, which can be approximated as

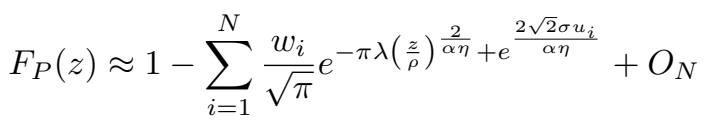

where, $O_{N}$ represents the remainder terms that decrease to zero as $N$ increases to infinity. By differentiating (11) with respect to $z$, the PDF of the transmit power $P$ can be obtained as given in (3). 


\section{APPENDIX B}

The uplink coverage probability, $P_{c}=\operatorname{Pr}[S I N R>T]$, can be derived as follows.

$$
\begin{aligned}
& P_{c} \stackrel{a}{=} \operatorname{Pr}\left[g>\frac{T\left(I_{\Phi \backslash x}+N_{0}\right)}{\rho r_{x}^{\alpha(\eta-1)}}\right] \\
& \stackrel{b}{\approx} 2 \pi \lambda \int_{0}^{\infty} r_{x} e^{-\frac{T N_{0} r_{x}^{\alpha(1-\eta)}}{\rho}-\pi \lambda r_{x}^{2}} \mathbb{E}_{I_{\Phi \backslash x}}\left[e^{-\frac{I_{\Phi \backslash x} T r_{x}^{\alpha(1-\eta)}}{\rho}}\right] d r_{x}
\end{aligned}
$$

where $r_{x}=\|x\|$ and $I_{\Phi \backslash x}=\sum_{z \in \Phi \backslash x} P_{z}\|z\|^{-\nu} h_{z}$ is the total interference power received by the BS located at the center of $\mathbb{R}^{2}$. Step (a) follows from (4). Step (b) is due to $g \sim \exp (1)$ and using the PDF of $r_{x}$ given in (2). Using the definition of the Laplace transform $\mathcal{L}_{X}(s)=\mathbb{E}\left[e^{-s X}\right]$, we can obtain the desired result given by (6).

\section{APPENDIX C}

Using the definition of the Laplace transform, $\mathcal{L}_{I_{\Phi \backslash x}}(s)=$ $\mathbb{E}\left[\exp \left(-s \sum_{z \in \Phi \backslash x} P_{z}\|z\|^{-\nu} h_{z}\right)\right]$. Therefore,

$$
\begin{aligned}
& \mathcal{L}_{I_{\Phi \backslash x}}(s)=\mathbb{E}_{\Phi \backslash x}\left[\prod_{z \in \Phi \backslash x} \mathbb{E}_{P_{z}, h_{z}}\left[\exp \left(-s P_{z}\|z\|^{-\nu} h_{z}\right)\right]\right] \\
& \quad \stackrel{a}{=} \exp \left(-2 \pi \lambda \int_{r_{x}}^{\infty}\left(1-\mathbb{E}_{P_{z}, h_{z}}\left[\exp \left(-s P_{z} r_{z}^{-\nu} h_{z}\right)\right]\right)\right) r_{z} d r_{z}
\end{aligned}
$$

where step (a) follows from the definition of probability generating functional of PPP [4]. $r_{z}=\|z\|$. Consider, $\mathcal{I}=$ $\left(1-\mathbb{E}_{P_{z}, h_{z}}\left[\exp \left(-s P_{z} r_{z}^{-\nu} h_{z}\right)\right]\right)$. Using the fact that $h_{z}$ and $P_{z}$ are statistically independent and substituting $f_{h_{z}}(h)$ given by (5)

$$
\mathcal{I}=1-\mathbb{E}_{P_{z}}\left[\int_{0}^{\infty} \frac{1}{1+\mu s P_{z} r_{z}^{-\nu}} \frac{e^{-\frac{(\ln (\mu))^{2}}{2 \xi^{2}}}}{\sqrt{2 \pi} \xi \mu} d \mu\right]
$$

Using change of variable $t=\frac{\ln \mu}{\sqrt{2} \xi}$

$$
\mathcal{I}=1-\mathbb{E}_{P_{z}}\left[\frac{1}{\sqrt{\pi}} \int_{-\infty}^{\infty} \frac{e^{-t^{2}}}{1+s P_{z} r_{z}^{-\nu} \exp [\sqrt{2} \xi t]} d \mu\right]
$$

Using the Gauss-Hermite quadrature in numerical analysis to circumvent the integration in (15), $\mathcal{I}$ can be written as

$$
\mathcal{I}=1-\mathbb{E}_{P_{z}}\left[\sum_{j=1}^{M} \frac{\beta_{j}}{\sqrt{\pi}} \frac{1}{1+s P_{z} r_{z}^{-\nu} \exp \left[\sqrt{2} \xi t_{j}\right]}\right]+O_{M}
$$

where $O_{M}$ is a remainder term, which decreases to zero as $M$ increases. $t_{j}$ and $\beta_{j}$ are abscissas and weight factors for the Hermite integration. Using the fact that $\sum_{j}^{M} \beta_{j}=\sqrt{\pi}$

$$
\mathcal{I} \approx \mathbb{E}_{P_{z}}\left[\sum_{j=1}^{M} \frac{\beta_{j}}{\sqrt{\pi}}\left(1-\frac{1}{1+s P_{z} r_{z}^{-\nu} \exp \left[\sqrt{2} \xi t_{j}\right]}\right)\right]
$$

Using the PDF of $P_{z}$ given in (3), $\mathcal{I}$ can be written as

$$
\mathcal{I} \approx \frac{2 \lambda}{\rho^{\frac{2}{\alpha \eta}} \alpha \eta} \sum_{i=1}^{N} w_{i} \sum_{j=1}^{M} \beta_{j} \int_{0}^{\infty} e^{\frac{2 \sqrt{2} \sigma u_{i}}{\alpha \eta}} \frac{P_{z}^{\frac{2}{\alpha \eta}-1}}{1+\frac{r_{z}^{\nu}}{s P_{z} \exp \left(\sqrt{2} \xi t_{j}\right)}}
$$

$$
\times \exp \left(\frac{-\pi \lambda P_{z}^{\frac{2}{\alpha \eta}} e^{\frac{2 \sqrt{2} \sigma u_{i}}{\alpha \eta}}}{\rho^{\frac{2}{\alpha \eta}}}\right) d P_{z}
$$

Using the change of variable $x=-\pi \lambda P_{z}^{\frac{2}{\alpha \eta}} e^{\frac{2 \sqrt{2} \sigma u_{i}}{\alpha \eta}} \rho^{\frac{-2}{\alpha \eta}}$

$$
\mathcal{I} \approx \frac{1}{\pi} \sum_{i=1}^{N} w_{i} \sum_{j=1}^{M} \beta_{j} \int_{0}^{\infty} \frac{e^{-x}}{1+\frac{r_{z}^{\nu}(\pi \lambda)^{\frac{\alpha \eta}{2}} \exp \left(\sqrt{2} \sigma u_{i}\right)}{s \rho \exp \left(\sqrt{2} \xi t_{j}\right) x^{\frac{\alpha \eta}{2}}}} d x
$$

The integral in (19) can be approximated as a Gauss-Laguerre quadrature sum as given below

$$
\mathcal{I} \approx \sum_{i=1}^{N} \frac{w_{i}}{\pi} \sum_{j=1}^{M} \beta_{j} \sum_{k=1}^{L} \kappa_{k} \frac{1}{1+\frac{r_{z}^{\nu}(\pi \lambda)^{\frac{\alpha \eta}{2}} \exp \left(\sqrt{2} \sigma u_{i}\right)}{s \rho \exp \left(\sqrt{2} \xi t_{j}\right) x_{k}^{\frac{\alpha \eta}{2}}}}+O_{L}
$$

where $x_{k}$ and $\kappa_{k}$ are the abscissas and weight factors for the Gauss-Laguerre integration [11, Table (25.9)] and $O_{L}$ is the remainder term, which reduces to zero as the number of terms $L$ increases. Substituting (20) in (13) gives the desired result in (7).

\section{ACKNOWLEDGEMENT}

Funding for this work has been provided by the Natural Sciences and Engineering Research Council (NSERC) of Canada, the Rohit Sharma Professorship and the Telecommunications Research Laboratories.

\section{REFERENCES}

[1] A. Goldsmith, Wireless Communications. Cambridge University Press, New York, 2005.

[2] M. Chiang, P. Hande, T. Lan, and C. W. Tan, Power control in wireless cellular networks, Foundations and Trends in Networking. NOW Publishers, Hanover, MA, Apr. 2008.

[3] H. ElSawy and E. Hossain, "Analysis of uplink transmissions in cellular networks: A stochastic geometry approach," in Proc. IEEE International Conference on Communications (ICC 2014), Jun. 2014, pp. 5783-5789.

[4] S. N. Chiu, D. Stoyan, W. Kendall, and J. Mecke, Stochastic Geometry and Its Applications, 3rd ed. John Wiley and Sons, 2013.

[5] M. Haenggi, Stochastic Geometry for Wireless Networks. Cambridge University Press, New York, 2013.

[6] T. Novlan, H. Dhillon, and J. Andrews, "Analytical modeling of uplink cellular networks," IEEE Trans. Wireless Commun., vol. 12, no. 6, pp. 2669-2679, Jun. 2013.

[7] H. ElSawy and E. Hossain, "On stochastic geometry modeling of cellular uplink transmission with truncated channel inversion power control," IEEE Trans. Wireless Commun., vol. 13, no. 8, pp. 4454-4469, Aug. 2014.

[8] V. Chandrasekhar and J. G. Andrews, "Uplink capacity and interference avoidance for two-tier femtocell networks," IEEE Trans. Wireless Commun., vol. 8, no. 7, pp. 3498-3509, Jul. 2009.

[9] H. Dhillon, R. Ganti, F. Baccelli, and J. Andrews, "Modeling and analysis of $\mathrm{K}$-tier downlink heterogeneous cellular networks," IEEE $J$. Sel. Areas Commun., vol. 30, no. 3, pp. 550-560, Apr. 2012.

[10] J. Andrews, F. Baccelli, and R. Ganti, "A tractable approach to coverage and rate in cellular networks," IEEE Trans. Commun., vol. 59, no. 11, pp. 3122-3134, Nov. 2011.

[11] M. Abramowitz and I. A. Stegun, Eds., Handbook of Mathematical Functions: with Formulas, Graphs, and Mathematical Tables. Dover Publications, New York, 1965.

[12] G. L. Stuber, Principles of Mobile Communication, 3rd ed. Springer, New York, 2011. 